\title{
PADRÕES AUTO-ORGANIZADOS, REPETITIVOS E SUPERPOSTOS DE MARCAS DE ONDULAÇÃO, DUNAS E DRAAS: UM ARRANJO DE GEOMETRIA FRACTAL?
}

\author{
ABÍLIO C.S.P. BITTENCOURT* \& OLIVAR A.L. LIMA*
}

\begin{abstract}
SELF-ORGANIZED, REPETITIVE AND SUPERIMPOSED PATTERNS OF RIPPLES, DUNES AND DRAAS: AN ARRANGEMENT OF FRACTAL GEOMETRY? A critical summary regarding the spatial and genetic relationship between ripples, dunes and draas developed on ergs in presented. It is proposed that these aeolian bedforms follow a geometrical fractal arrangement at different scales, in response to the chaotic atmospheric circulation system responsible for their origin. A geometrical model is presented to describe the relation between number of features, its average dimension and the fractal dimension for the set This relation may be useful for describing and representing actual geomorphic features giben their relative dimensions and numbers. It is also discussed the possibility that such features may reflect a statistically fractal development in time. If this is the case, it would be possible to predict, on a wide time scale, the future development of ergs or, going back in time, to set a geochronology for them.
\end{abstract}

Keywords: Fractal geometry, chaotic system, erg, aeolian bedforms.

\begin{abstract}
RESUMO É apresentado um sumário crítico a respeito das relações espaciais e genéticas entre marcas de ondulações, dunas é draas dessenvotvidas em ergs. Propõe-se que estas formas de leito eólicas guardam entre si, em diferentes escalas, um arranjo geométrico fractal, em resposta ao sistema caótico de circulação atmosférica que as geram. Um modelo geométrico é apresentado paara descrever a relação entre o número de feições, suas dimensões médias e a dimensão fractal para cada conj unto. Esta relação pode ser útil para descrever e representar as características geomórficas atuais de tais feições, fornecendo os seus números e dimensões relativas. É também discutida a possibilidade de que tais feições possam refletir um desenvolvimento estatisticamente fractal no tempo. Se este for o caso, será possível predizer, em vasta escala de tempo, o desenvolvimento futuro dos ergs ou, recuando no tempo, estabelecer uma geocronologia para os mesmos.
\end{abstract}

Palavras-chave: Geometria fractal, sistema caótico, erg, formas de leito eólicas.

INTRODUÇÃO A principal característica da geometria fractal é a auto-similaridade: um padrão de feições repetitivas que é invariável na escala (Goupillaud 1991). As características morfológicas observadas numa determinada escala podem ser vistas em outras escalas. Um exemplo elucidativo de geometria fractal, citado em Goupillaud (1991), pode ser visto na hidrodinâmica de um rio. A velocidade de partículas do meio líquido, combinada à rugosidade do leito do rio, produz um fluxo turbulento que induz a formação de redemoinhos auto-similares, numa ampla gama de dimensões, variando desde rotações em grande escala até pequenos vórtices que, no fim, dissipam a energia cinética do fluido.

A geometria fractal é gerada por sistemas dinâmicos nãolineares (Middleton 1991), escapando assim das características de continuidade e suavidade, que são atriburos da geometria euclidiana (Goupillaud 1991). Na natureza, são inúmeros os exemplos de sistemas dinâmicos não lineares geradores de padrões fractals que podem ser ordenados tanto no espaço quando no tempo (Turcotte 1990). Dessa maneira, muitos objetos naturais podem ser representados usando fractais, tais como linhas costeiras, nuvens, folhas e plantas ramificadas, por exemplo. Na área das Geociências, muitos são os exemplos de comportamentos fractais: a. distribuição dos tamanhos de grãos e de poros em sistemas particulados (Turcotte 1986); b. relação entre o número de terremotos e a dimensão linear da zona de ruptura (Hsü 1990); c. distribuição dos tamanhos de corpos de minérios (Middleton 1991); d. relação entre o número de discordancies e os períodos de deposição (Plotnick 1986), entre outros. E bem significativo o exemplo apresentado por Stilley (1991), enfatizando a aparência regular de formas repetitivas observáveis em um vasto alcance de escalas no campo da tectônica, desde estruturas locais, através de sub-bacias, baciais, continentais até extracontinentais. Ademais, o referido autor considerou essas formas, auto-similares, como manisfestações de um sistema dinâmico não-linear, que opera no interior do planeta.

Em relação à auto-similaridade e ao alcance das escalas de observações de feições fractais, deve-se levar em conta, como aponta Hsü (1990), que os fenômenos naturais têm limites de magnitude. Assim, a auto-similaridade é satisfeita somente dentro de uma gama finita de escalas. Por exemplo, como menciona Turcotte (1990), do tamanho do corpo que é fragmentado, até o tamanho de um grão. Da mesma forma, não se pode deixar de levar em conta que fractais exatamente auto-similares são entidades matemáticas não encontradas na natureza (Fowler \& Roach 1991). Portanto, os objetos fractais da natureza podem não aparecer invariantes ao se expandir ou reduzir a escala além de certos limites (Gleick 1990, Goodings \& Middleton 1991).

Um dos mais marcantes e notórios padrões repetitivos de formas que se apresentam em diferentes escalas, na natureza, é encontrado nos ergs (campos de dunas), consubstanciado em formas de leito de tamanhos diversos (draas, dunas e marcas de ondulações), que se superpõem umas às outras (Fig. 1). A ubiqüidade e regularidade de tais padrões geomórficos são largamente reconhecidas em toda a literatura sobre o assunto. Neste trabalho, é promovida uma revisão visando a descrição dos parâmetros espaciais mais significativos dessas feições, bem como de seus relacionamentos de escala usando a auto-similaridade de um modelo de geometria fractal. As relações obtidas parecem reproduzir satisfatoriamente alguns dos parâmetros dimensionais observados em sistemas de ergs do planeta, dependendo do uso de uma dimensão fractal apropriada. Á aplicabilidade do modelo, 


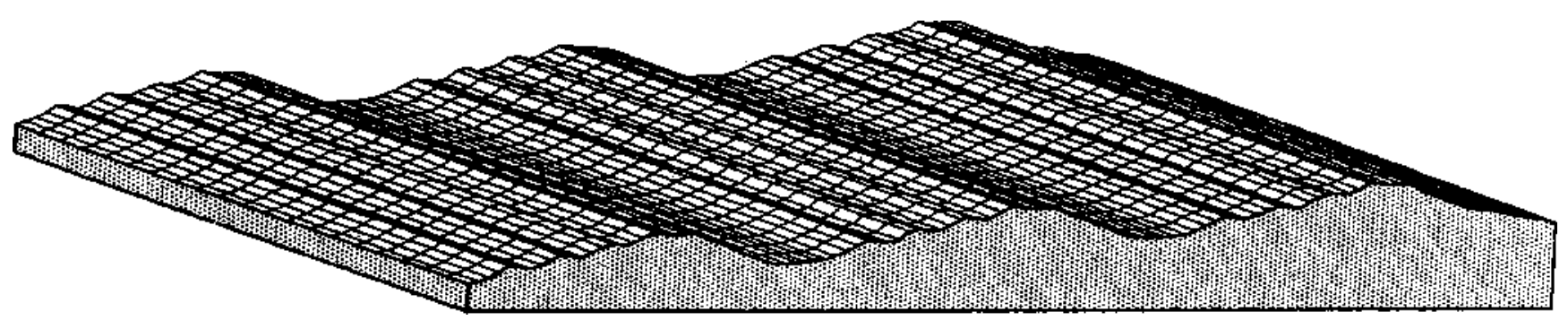

Figura 1 - Modelo esquemático mostrando os padrões repetitivos e superpostos de marcas de ondulação e dunas sobre

Figure l - Schematic model showing the repetitive and superimposed patterns of tipples and dunes over a draa

todavia, necessita ser melhor tratada através de uma análise mais detalhada da geometria de superposição das várias feições em campos de ergs específicos.

\section{DRAAS, DUNAS E MARCAS DE ONDULAÇÃO - RELAÇÕES ESPACIAIS Um dos principais} trabalhos a primeiro chamar a atenção para a marcante regularidade observada nos padrões de distribuição das formas de leito eólicas presentes nos ergs foi o de Bagnold (1941).Esse autor, referindo-se ao espaçamento entre as cristas de dunas presentes em diferentes ergs por ele estudados em diversas partes do mundo, comenta, vivamente impressionado que... "the width is approximately the same over a large area,sothat the chains, or, on the largest scalethe multiple dune systems, all lie parallel to one another and are evenly spaced, thus giving the country an extraordinary aspect of geometrical order unseen elsewhere innature except on a microscope". Posteriormente, diversos autores descrevem sobre tal aspecto dos ergs, como, por exemplo, McKee (1966), Norris (1965) Norris \& Norris (1961), Inman et al. (1966) e Wilson (1972a, b, 1973), enquanto Hallet (1990) reconhece possuírem essas feições geomórficas um caráter de auto-organização.

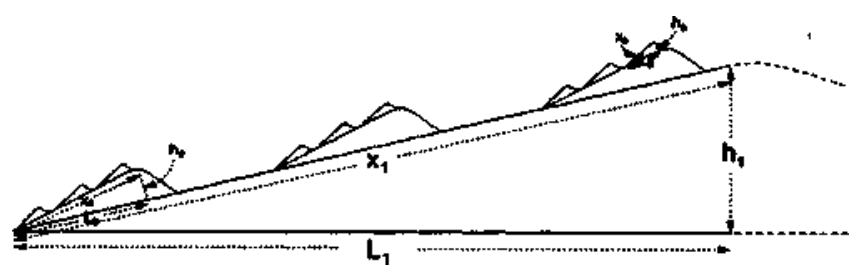

Figura 2 - Campos de dunas do tipo self. A. Austrália; B. Libia (segundo Wilson 1972a, Pranchas IIIC e D, p. 183) Figure 2 - Dune fields of the seif type. A. Australia; B. Lybia (aflter Wilson 1972a, Charts IIIC and D, p. 183)

Tais padrões geométricos são observáveis em diferentes partes do mundo. Assim, nos ergs da Austrália, bem como nas partes dos ergs do norte da Africa, nos quais predominam dunas do tipo seif (lineares), Bagnold (1941) observou marcante similaridade na aparência entre mapas e fotosaéreas de ambas as áreas. Essas regiões são caracterizadas por cadeias estreitas, paralelas e regularmente espaçadas de seif que, segundo esse autor, tornam quase indistinguíveis as fotos aéreas e mapas entre uma região e outra. Campos de seif com feições semelhantes podem também ser observados nas figuras $2 \mathrm{~A}$ e B. Padrões parecidos, embora não tão notáveis quanto em relação às dunas do tipo seif, podem também ser constatados em campos de barcanas, que se apresentam na forma de cristas sinuosas regularmente espaçadas (Fig. 3 ). Na costa brasileira, pode ser citado o exemplo do erg localizado na planície costeira associada à desembocadura do Rio São Francisco. Ali, embora as dunas estejam muito próximas da fonte alimentadora de areia - situação que, segundo Bagnold (1941) e Norris \& Norris (1961), condiciona um empacotamento entre as mesmas - ainda assim, apresenta-se na forma de enxames de barcanas vagamente delineadas paralelamente ao corpo principal do erg (Fig. 4). Além das dunas, da mesma maneira, as duas outras formas de leito eólicas que se sobressaem nos ergs, marcas de ondulação e draas, também apresentam os mesmo padrões repetitivos. Quanto às marcas de ondulação, que se deslocam sobre as dunas, como é de sobejo conhecido, vasta é a literatura sobre o assunto. Em relação aos draas, sobre os quais se deslocam as dunas, bons exemplos podem ser vistos na figura 5 . Segundo Wilson (1973), os elementos geomórficos acima mencionados (marcas de ondulação, dunas e draas) constituem os três grandes grupos universalmente descontínuos, isto é, sem formas intermediárias entre os mesmos, em que podem ser divididas as formas de leito eólicas. Nesse sentido, eles podem ser aproximadamente distinguidos pelo comprimento de onda, da seguinte maneira: draas - de 1 a $3 \mathrm{~km}$, dunas - comumente de 15 a $250 \mathrm{~m}$, e marcas de ondulação comumente de 1 a $20 \mathrm{~cm}$. Usualmente, essas formas de leito ocorrem simultaneamente, mantendo o mesmo padrão repetitivo (Wilson 1972a). Esses padrões geomórficos, como ressalta Bagnold (1941), mantém-se em diferentes circunstâncias, independentemente das características locais, como é o caso dos mesmos serem formados tanto em: a. ergs nãodestituídos de vegetação, como os que são encontrados na Austrália, quanto naqueles em que a mesma inexiste, como no Egito; b. em ergs jovens, como ainda os da Austrália, se comparados aos do Egito, bem antigos. Esse fato sugere que essas formas de leito são fenômenos espontâneos universais e relacionados entre si, com origens similares (Wilson 1972a) Nesse sentido, Wilson (1972b, Fig. 2, p. 668) apresenta um gráfico que relaciona os comprimentos de onda associados às formas de leito, no sentido longitudinal e transversal às mesmas, demonstrando que os mesmos estão bastante próximos um do outro numa vasta gama de valores (de $0,5 \mathrm{~cm}$ a 6 $\mathrm{km}$ ), o que enfatiza o fato de que deve haver alguma espécie de ligação causal entre os mecanismos geradores dessas formas de leito.

O padrão de disposição das formas de leito eólicas em um erg pode ser um simples arranjo de cristas paralelas ou, mais usualmente, uma rede de dois ou três sistemas de cristas que se interceptam em ângulos distintos, formados por regimes de ventos multidirecionais (Wilson 1972b). Esses trends individuais de dunas e draas, freqüentemente, como aponta Wilson (1973), podem ser seguidos por diversas centenas de quilômetros através dos ergs, e de um erg para outro, o que importa dizer que eles são regionalmente significativos, não podendo ser atribuídos a efeitos locais ou ao acaso.

Por fim, deve-se ressaltar que interessantes resultados foram obtidos por Wilson (1973), a partir de medidas efetuadas nos draas existentes em três diferentes ergs da Argélia, completamente recobertos de areia. Assim, a partir de draas apre- 

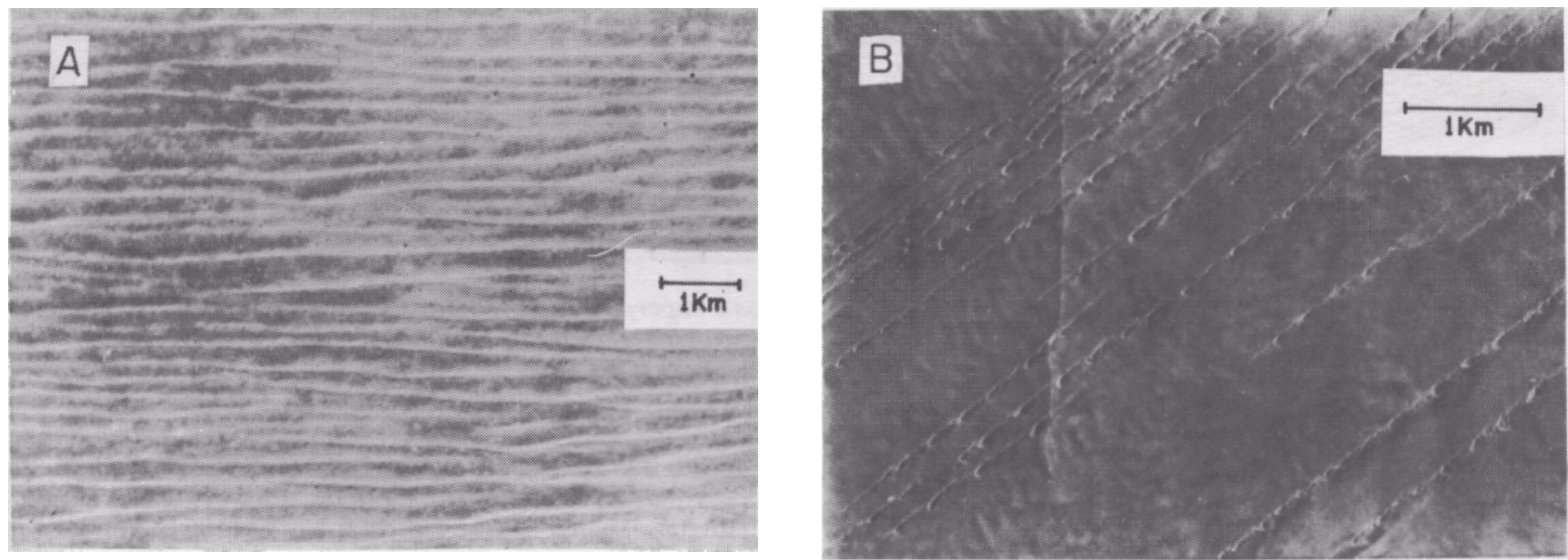

Figura 3 - Campos de barcanas no México (segundo Inmann et al. 1966, Prancha 1, p. 792)

Figure 3 - Barchans field in Mexico (after Inmann et al. 1966, Chart 1, p. 792)

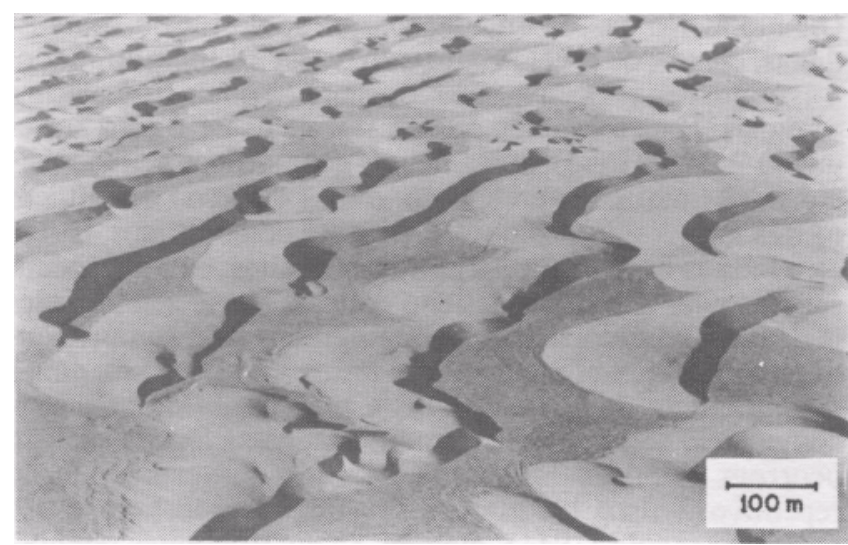

Figura 4 - Campo de barcanas da planície costeira associada à desembocadura do Rio São Francisco (segundo Dominguez et al. 1991, Fig. 9B)

Figura 4 - Barchans field at the coastal plain associated to the São Francisco river mouth (aflter Dominguez et al. 1991, Fig. 9B)

sentando alturas variando entre 10 e $400 \mathrm{~m}$ e comprimentos de onda entre 0,5 e 5 km, Wilson (1973, Fig. 10, p. 94) construiu um gráfico que mostra uma boa correlação positiva entre essas dimensões.

MODELO FRACTAL DAS FORMAS DE LEITO EOLICAS A mais importante característica quantitativa de um objeto fractal é a sua dimensão fractal. A figura 6 apresenta um perfil esquemático de uma sucessão de formas de leitos eólicas usado na análise geométrica de geração e superposição dessas estruturas. Essas figura representa um único draa, cuja altura é bem menor do que sua dimensão longitudinal. Com isso, é válida a seguinte aproximação $\mathrm{x}_{1} \mathrm{~L}_{1}$, sendo $\mathrm{x}$, o comprimento da face a barlavento e $\mathrm{L}_{1}$ a dimensão de sua pro ' ' ' ' Nontal. No caso mais geral, deve-se ter $X_{1}=v \boldsymbol{L}_{1}+\boldsymbol{r}_{1}$, onde $\mathrm{h}$, é a altura do draa (Fig. 6).

Sobre o draa desenvolve-se uma seqüência de duans construídas de modo a garantir a validade da aproximação dimensional $\mathrm{L}_{2}$. Além disso, pode-se assumir com boa aproximação q'ue as cristas das dunas na seqüência estão separadas entre si por espaços interdunares com dimensões de mesma grandeza $\mathrm{L}_{2}$. Na face, a barlavento de cada duna,

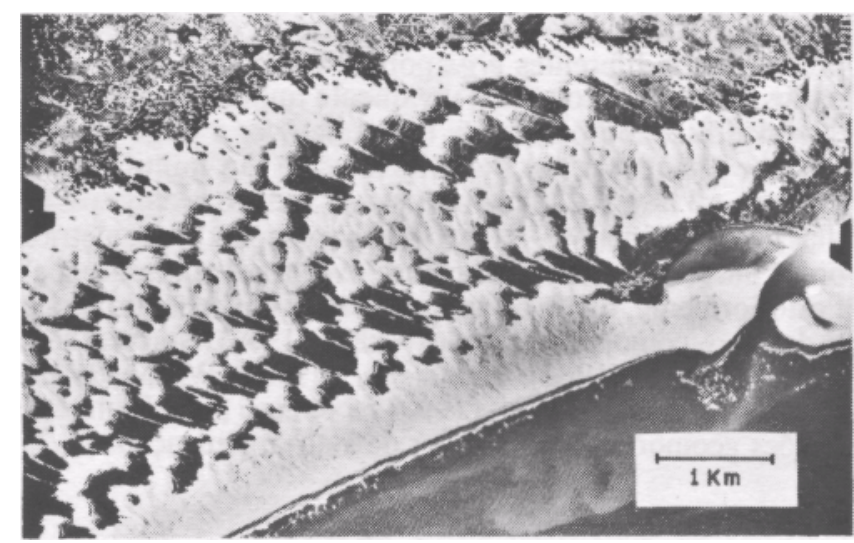

Figura 5 - Dois diferentes campos de Draas do tipo barcanóide. O enrugamento na superfície dos draas é provocado por dunas superpostas (segundo Wilson 1972a, pranchas IID e E, $p .178$ e 179)

Figure 5 - Two different draas fields of barchanoid type. The wrinkled aspect on the draa surface is caused by the superposition of many dunes (after Wilson 1972a, tables IID and E, p. 178 and 179)

desenvolvem-se marcas de ondulação, nas quais, por similaridade, propõe-se a ação da mesma regra de reprodução da geometria desses leitos.

Dessa maneira, em cada geração de feições eólicas, a partir do draa, é válida a seguinte expressão relacionando a dimensão da feição $\left(\mathrm{L}_{\mathrm{i}+1}\right)$ e o número de feições $\left(\mathrm{N}_{\mathrm{i}+1}\right)$ que estarão sobrepostas à feição geométrica da família antecedente $\left(\mathrm{L}_{\mathrm{i}+\mathrm{Ni}}\right)$ :

$$
N_{i+1}=N_{i}\left(\frac{L_{i}}{L_{i+1}}\right)^{D} ; i=1,2
$$

A equação (1) é obtida utilizando-se a equação de definição de dimensão fractal de uma forma geométrica (Barnsley 1988). A geometria criada segundo o procedimento descrito possui dimensão de Hausdorff dada por:

$$
D=\frac{\log \left(N_{i+1} / N_{i}\right)}{\log \left(L_{i} / L_{i+1}\right)}
$$



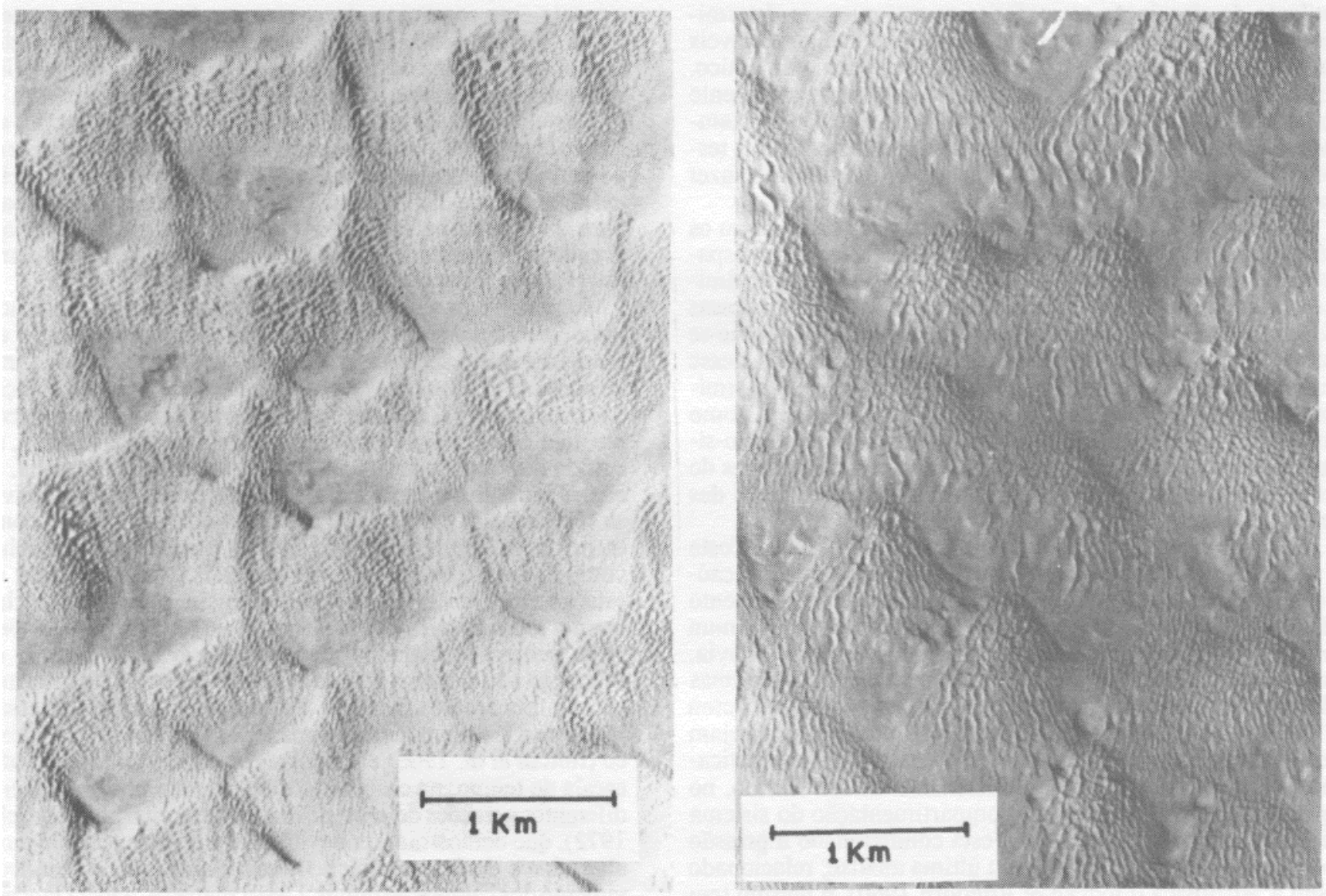

Figura 6 - Perfil esquemático de um erg mostrando as dimensões das marcas de ondulações, dunas e draas utilizadas para o calculo da dimensão fractal dessas feições geomórficas. O número de feições superpostas à anterior de maior hierarquia é meramente ilustrativo, não representando, portanto, aquele que é gerado fractalmente pelo sistema

Figura 6 - Schematic profile of an erg showing the dimensions of ripples, dunes and draas used to compute the fractal dimension of these geomorphic features. The number of features in each family is only illustrative and do not represent the actually generated by the system

As expressões (1) e (2) podem ser ligeiramente modificadas para descrever o caso mais geral no qual $\mathrm{x}, \neq \mathrm{L}_{1} \mathrm{e} / \mathrm{ou}$ quando a distância entre a crista de uma feição e a da sua contígua for menor, mas ainda proporcional a sua dimensão horizontal.

DRAAS, DUNAS,E MARCAS DE ONDULAÇÃO RELAÇÕES GENETICAS Para que um erg apresente um padrão de draas, dunas e marcas de ondulação coexistentes, é preciso que o mesmo seja antigo e, portanto, perfeitamente desenvolvido. Efetivamente, os padrões de draas requerem um considerável tempo para se desenvolver (de 5.000 a 1.000 .000 anos para crescer completamente, segundo Wilson 1971). Ademais, como menciona Wilson (1973), é necessário uma certa abundância de areia no erg, para que existam condições de se formarem núcleos adequados, na forma de grandes dunas superpostas, para o crescimento dos draas. Nessas condições, o desenvolvimento dos padrões das formas de leito é completo, isto é, as mesmas podem ser subdivididas em células unitárias similares repetidas em intervalos regulares (Wilson 1972a), como pode ser percebido na figura 5, em relação aos draas. Em contrapartida, num padrão com desenvolvimento incompleto, as formas de leito estão nucleadas somente em parte, de tal maneira que diferentes estágios de desenvolvimento podem ser encontrados em partes diversas, não tendo tido ainda as mesmas tempo suficiente para interagir lateralmente e ajustar os seus espaçamentos (Wilson 1972a).

Um processo gerador de uma geometria de circulação fractal semelhante ao que acontece durante o fluxo turbulento de um rio é também verificado no fluxo turbulento do ar sobre um leito arenoso. Padrões de redemoinhos de diferentes dimensões são manisfestações das características caóticas que são atribuídas ao sistema atmosférico por Turcotte (1990). Como considera Hallet (1990), vórtices espiralados de fluxo são gerados espontaneamente devido às fortes interações e realimentações diretas entre o fluxo, o transporte de sedimentos e a morfologia do leito granular na base do fluxo. Quando o fluxo de ar é arrastado contra o solo, existem muitas situações onde fluxos secundários podem se desenvolver, caracterizados em células regularmente repetidas com variações de velocidade superpostas às variações do fluxo principal (Wilson 1972a). Segundo Wilson (1972b), as marcas de ondulações, dunas e draas são provavelmente causadas pela ação desses fluxos secundários em células regulares de diferentes escalas no vento, próximo ao chão.

DISCUSSÃO E CONCLUSÕES Tendo em vista o que foi analisado nos itens anteriores, as marcas de ondulações, dunas e Draas podem ser consideradas como eventos analisáveis (Stilley 1991) do sistema dinâmico de circulação atmosférica, no sentido de que representam um sumário das necessidades mecânicas requeridas para formá-las. As dimensões dessas feições geométricas criadas pelo vento em fluxo turbulento devem assim guardar relações proporcionais simples com as dimensões das células circulatórias de ar que as produziram. Elas expressam, assim, o resultado físico da ordenação coerente com que a energia do fluxo tur- 
bulento da circulação atmosférica se estrutura em determinadas circunstâncias e representam uma das mais notáveis manifestações de geometria fractal desse sistema dinâmico. Evidentemente, simples modelos matemáticos raramente produzem um perfeito ajuste com o mundo real e isto também é verdadeiro para os modelos fractais. Dessa forma, testes de validade das equações deste trabalho deverão fazer parte de estudos posteriores.

Uma das possíveis conseqüências de se assumir serem os draas, dunas e marcas de ondulações manifestações espaciais de uma geometria fractal associada ao sistema dinâmico da circulação atmosférica é que o desenvolvimento dessas formas de leito e, conseqüentemente, dos ergs, comporte-se também fractalmente no tempo. Mais provavelmente, nesse caso, não existirá uma relação fractal exatamente auto-similar mas, sim, do tipo que Turcotte (1990) considera como auto-afim, ou, em outras palavras, estatisticamente auto-similar (Fowler \& Roach 1991); isto é, as pequenas partes do sistema mostram as mesmas propriedades estatísticas das partes maiores.

Evidentemente, como argumentam Pires \& Vaz da Costa (1992) e Grebogi et al (1987), a evolução dos sistemas caóticos, em virtude das incertezas relativas ao conhecimento das condições iniciais de suas gerações, não é previsível num sentido determinístico para períodos muito longos. Todavia, essa sensível dependência às condições iniciais dos sistemas dinâmicos não-necessariamente, como observa Middleton (1991), implica que os estados futuros dos mesmos sejam totalmente desconhecidos, podendo ser tratados estatisticamente como mencionado anteriormente. Nesse sentido, no que tange ao padrão geral de compartimentação do sistema de circulação atmosférica, que está condicionado à pressão constante da energia solar e, em última análise, relacionado com a astrofísica (Opdyke \& Rundorn 1960), o mesmo tem mantido por muito tempo as suas características gerais. Como um exemplo, cite-se o caso das cinturões de ventos alísios, que existem pelo menos desde o Paleozóico, como pode ser inferido a partir de seqüências desérticas paleozóicas existentes no Platô do Colorado, EUA (Bigarella 1972). Esse padrão geral global da circulação atmosférica deverá manter-se ainda por muito tempo. Assim, embora o movimento dos corpos celeste revele também uma natureza caótica (vide a distribuição fractal dos meteorites e asteróides - Turcotte 1990), o sistema solar pode ser considerado como estável pelo mesnos por um bilhão de anos ainda (Pires \& Vaz da Costa 1992). Dessa maneira, parece inteiramente razoável a perspectiva de se poder vir a fazer previsões estatisticamente fractais, em escalas de tempo bem amplas, em relação ao desenvolvimento dos ergs que, como já se disse está funda- mentalmente condicionado ao sistema de circulação atmosférica. Tais previsões, todavia, não devem desconhecer os distúrbios antropogênicos produzidos sobre os fluxos de energia e materiais, que poderão, de alguma forma, comprometê-las.

Nas circunstâncias acima descritas, a relação fractal, segundo Hsü (1990), implica que o intervalo de tempo decorrido entre dois eventos da mesma magnitude seja proporcional à magnitude dos eventos. Ter-se-á, dessa forma, uma base para extrapolar as estatísticas disponíveis em relação aos eventos de pequena escala de tempo, a fim de se estimar o intervalo de recorrência dos grandes eventos.

Tomando como exemplo os ergs existentes na costa norte-nordeste brasileira, é possível que exista uma relação estatisticamente fractal entre os pulsos de migração das dunas atualmente aí ativas e as fases de desenvolvimento e fixação dos referidos ergs, durante p Quaternário. Sabe-se, por exemplo, que no erg atual costeiro, na região de Luís Correia, no Estado do Piauí, a migração das dunas, do tipo barcana, só ocorre durante a estação seca, sendo interrompida na chuvosa (Bittencourt et al. 1990). Nessas circunstâncias, como mencionam Dominguez et al. (1991), durante a estação chuvosa, as áreas interdunares são alagadas, vindo a secar na estação sem chuvas. Como conseqüêencia, esses autores chamam a atenção para o fato de que a região interdunar apresenta pequenas cristas arqueadas, côncavas na direção de migração das dunas, representando os períodos de interrupção no trânsito das mesmas. Feições similares, vale ressaltar, foram também descritas nos EUA (Norris 1965), México (Inman et al. 1966) e Tunísia (Purvis 1991). Já, em outra escala de tempo, na costa brasileira existem pelo menos três diferentes gerações de ergs, de idades quaternárias (Bigarella 1972), que demostram ter havido, durante esse período, uma alternância de fases propícias e inibidoras para o desenvolvimento dos campos de dunas. Dessa maneira, a idéia lançada como uma hipótese neste trabalho é de que seria possível, após a decodificaçãp das relações fractais existentes entre a freqüência das distribuições dos pulsos de migração das barcanas e as fases de aparecimento e desaparecimento desses ergs costeiros - sobre os quais já se dispõe de algumas indicações de idade nas regiões costeiras dos Estados da Bahia (Martin et al. 1979-1980), Sergipe (Bittencourt et al. 1983) e Alagoas (Barbosa et al. 1986) - dispor-se de um fio condutor para se fazer, de um lado, tanto previsões relativas ao desenvolvimento futuro dos ergs, quanto, de outro, recuando no tempo, uma geocronologia dos ergs antigos. A par disso, concomitantemente, esforços deveriam ser envidados no sentido de se tentar calibrar esta estatística fractal com os ciclos astrofísicos que, em última análise, induzem os ciclos clomáticos na Terra (Fairbridge 1970, Fisher 1986, Petit-Maire 1991).

\section{REFERÊNCIAS BIBLIOGRÁFICAS}

BAONOLD, R.A. 1941. The Physics of Blown Sand and Desert Dunes. London, Methnen. 265 p.

BARNSLEY, M. 1988. Fractals Everywhere. New York, Academic Press Inc. BARBOSA, L.M.; BITTENCOURT, A.C.S.P.; DOMINGUEZ, J.M.L. MARTIN, L. 1986. The Quaternary coastal deposits of the State of Alagoas: influence of the relative sea-level changes. In: RABASSA, J. ed. Quaternary of South America and Antarctic Peninsula. Balkema, Rotterdam, v. 4-A, p. 269-290.

BIGARELLA, J.J. 1972. Eolian environments: their characteristics, recognition and importance. In: RIGBY, J.K. \& HAMBLIN, W.K. eds. Recognition of Ancient Sedimentary Environments. London, SEPM. p. 12-62. (Special Publication 16).

BITTENCOURT, AC.S.P.; MARTIN, L.; DOMINGUEZ, J.M.L.; FERREIRA, Y.A. 1983. Evolução paleogeográfica quaternária da costa do Estado de Sergipe e da costa sul do Estado de Alagoas. Rev. Bras. Geoc., 13(2):9397.

BITTENCOURT, A.C.S.P.; DOMINGUEZ, J.M.L.; MOITA FILHO, 0.1990. Variações texturais induzidas pelo vento nos sedimentos da face da praia (Praia de Atalaia-Piauí). Rev. Bras. Geoc., 20(1-4):201-207.

DOMINGUEZ, J.M.L.; BITTENCOURT, A.C.S.P.; MARTIN, L. 1991. Controls on Quaternary coastal evolution of the east-northeast coast of Brazil: roles of sea-level history, trade wbds and climate. Sediment Geol., 80:213-232.
FAIRBRIDGE, R.W. 1970. World paleoclimatology of the Quaternary. Rev. Géogr. Phys. Géol. Dynamique, 12(2): 97-104.

FISCHER, A.G. 1986. Climate Rhythms recorded in Strata. Ann. Rev. Earth Planet. Sci., 14:351-376.

FOWLER, T. \& ROACH, D. 1991. Dimmensionality analysis of objects and series data. In: MIDDLETON, G. V. ed. Non linear Dynamics, Chaos and fractals (With Applications to Geological Systems). Canada, Geol. Assoc. Canada, p. 59-81. (Short Course Notes 9).

GLEICK, J. 1990. Caos: a Criação de uma Nova Ciência. Campus, Rio de Janeiro. $310 \mathrm{p}$.

GOODINGS, D. \& MIDDLETON, G. V. 1991. Fractals and fractal dimensioa In: MIDDLETON, G. V. ed. Non linear Dynamics, Chaos and fractals (With Applications to Geological Systems). Canada, Geol. Assoc. Canada. p. 13-22. (Short Course Notes 9).

GOUPILLAUD, P.L. 1991.The role of chaotic dynamics and fractal geometry in exploration. Geophysics: the Leading Edge of Exploration, 10:25 e 28-30.

GREBOGI, C.; OTT, D.; YORKE, J.A. 1987. Chaos, strange attractors, and fractal basin boundaries in nonlinear dynamics. Science, 238:632-638.

HALLET, B. 1990. Spatial self-organization in Geomorphology: from periodic bedforms and patterned ground to scale-invariant topography. Earth-Sci. Rev., 29:57-75.

HSU, K.J. 1990. Actualistic catastrophism and global change. Palaeogeogr., 
Palaeoclimatol, Paleoecol, 89:309-131.

INMAN, D.L.; EWING, G.C.; CORLISS, J.B. 1966. Coastal sand dunes of Guerrero Negro, Baja California, Mexico. Geol. Soc. Am. Bull., 77:787802.

MARTIN, L.; VILAS BOAS, G.S.; BITTENCOURT, A.C.S.P.; FLEXOR, J.M. 1979-1980. Origine dês sables et ages dês dunes de la region de Salvador (Bresil) - consequences paleoclimatiques. Cahier ORSTOM, XI (1): 125132. (Ser. Geol.)

MCKEE, E.D. 1966. Structures of dunes at White Sands National Monument, New Mexico (and a comparison with structures of dunes from other selected areas. Sedimentology, 7:1-70.

MIDDLETON, G.V. 1991. Introduction to nonlinear dynamics, chaos and fractals. In: MIDDLETON, G.V. ed. Non linear Dynamics, Chaos and fractals (With Applications to Geological Systems). Canada, Geol. Assoc. Canada, p. 1-12. (Short Course Notes 9).

NORR1S, R.M. 1965. Barchan dunes of Imperial valley, California. 7. Geol, 74:292-306.

NOR RIS, R.M. \& NORRIS, K.S. 1961. Algodones dunes of southeastern California. Geol. Soc. Am. Bull., 72:605-620.

OPDYKE, N.D. \& RUNCORN, S.K. 1960. Wind direction in the western United States in the Late Paleozoic. Geol. Soc. Am. Bull., 71:959-972.

PETIT-MAIRE, N. 1991. The role of Earth Science in the anticipation of future climatic change. Ciência e Cultura, 43:130-136.

PIRES, A.S.T. \& VAZ DA COSTA, B. 1992. A desordem inevitável. Ciência Hoje, 14:34-39.
PLOTNIK, R.E. 1986. A fractal model for the distribution of stratigraphic hiatuses. J. Geol., 94:885-890.

PURVIS, K. 1991. Stoss-side mud-drapes: deposits of interdune pond margins. Sedimentology, 38:153-156.

STILLEY, W.T. 1991. A geotectonic model for petroleum exploration Geophysics: The Leading Edge of Exploration, 10:23-27.

TURCOTTE, D.L. 1986. Fractals and fragmentation. J. Geophys. Res. 91:1921-1926.

TURCOTTE, D.L 1990. Implications of chaos, scale-invariance, and fractal statistics in geology. Palaeogeogr., Palaeoclimatol., Paleoecol., 89:301138.

WILSON, I.G. 1971. Desert sandflow basins and a model for the development ofergs.Geogr.J., 137:180-189.

WILSON, I.G. 1972a. Aeolian bedforms - their development and origins. Sedimentology., 16:173-210.

WILSON, I.G. 1972b. Universal discontinuities in bedforms produced by the wind. /. Sediment. Petrol., 42:667-669.

WILSON, I.G. 1973. Ergs. Sediment. Geol., 10:77-106.

MANUSCRITO NP003

Recebido em 18 de Janeiro de 1993

Revisão do autor em 10 de maio de 1994

Revisão aceita em 20 de maio de 1994 